\title{
An unanticipated role
}

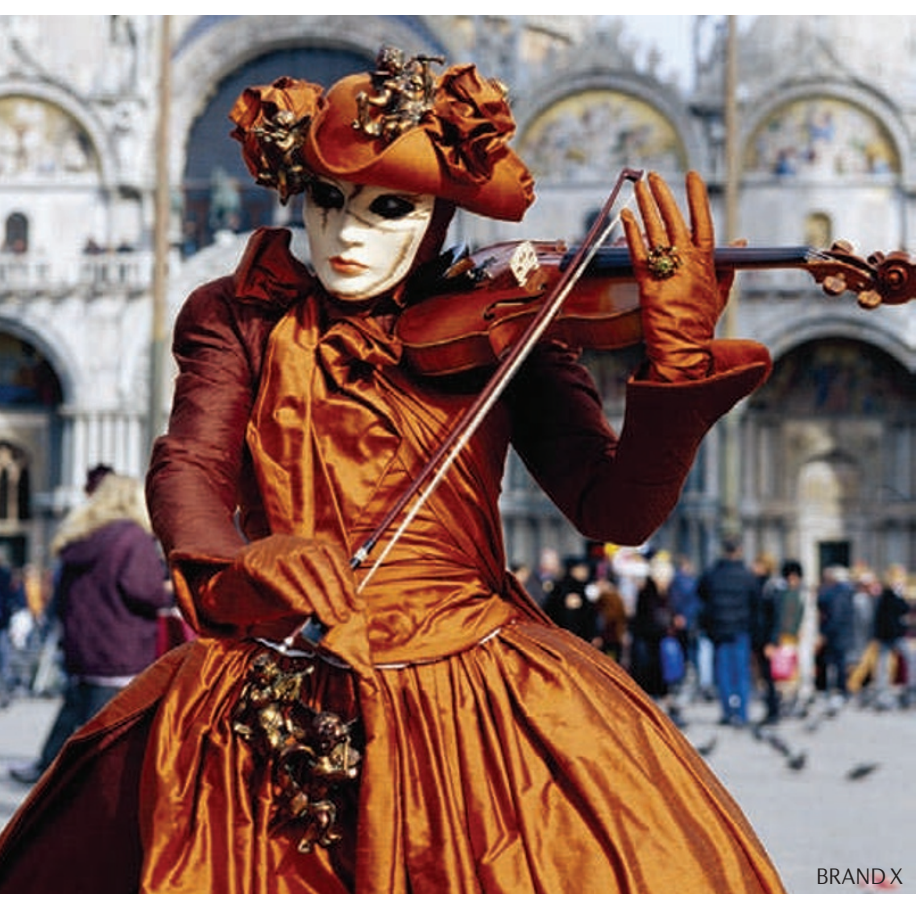

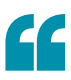

PcG proteins

can also

function

as tumour

suppressors

by repressing

key oncogenic

signalling

pathways.
Polycomb group (PcG) proteins silence differentiation genes during development and have been thought to have a primarily oncogenic role in human cancer. However, two studies in Drosophila melanogaster show that PcG proteins can also function as tumour suppressors by repressing key oncogenic signalling pathways.

Anne-Marie Martinez, Giacomo Cavalli and colleagues generated D. melanogaster larval clones that were homozygous knockouts for the PcG polyhomeotic ( $p h$ ) locus in eye imaginal discs. These imaginal discs were larger than controls, and the adults that emerged from the few surviving pupae had enlarged eyes with an abnormal morphology. Immunostaining revealed increased rates of mitosis, reduced differentiation and loss of normal polarity in the $p h$-null imaginal discs, indicating that $p h$ might be a bona fide tumour suppressor. Indeed, ph clone-derived tumours could be successively retransplanted into wild-type flies in which they were able to grow and metastasize, eventually leading to death. How does loss of ph trigger tumour development? The expression of Notch, one of its ligands, Serrate (Ser), and a downstream effector, eyegone (eyg), was upregulated in $p h$-null cells, and the expression of all these proteins is regulated by $\mathrm{PH}$. However, although overexpression of Notch in the D. melanogaster eye led to a phenotype that was similar to the loss of $p h$, inhibiting Notch signalling by overexpressing a dominant-negative form of Notch or by using RNA interference to repress Notch in a ph-null background produced flies with normal sized eyes but with an abnormal structure, suggesting that other PcG targets might have a role in determining the $p h$ mutant phenotype.

David Bilder and colleagues also used the D. melanogaster imaginal disc system to study PcG proteins. They identified and mapped a tumour suppressor mutant, the $P 3 C$ allele, showing that it is a deletion of two members of polycomb repressive complex 1 (PRC1): Posterior sex combs (Psc) and Suppressor of zeste $2(\underline{\mathrm{Su}}(z) 2)$. To investigate whether other PRC1 components also control the growth of imaginal discs, these authors examined the phenotypes of various PRC1 mutants and found that Polycomb $(\underline{P c}), p h$ and Sex combs extra ( $\underline{\text { Sce }})$ all caused the overgrowth of eye discs and led to pupal lethality. In these mutants, the Janus kinase (JAK)-signal transducer and activator of transcription (STAT) pathway ligand $u p d$ (also known as outstretched) and its paralogues $\underline{u p d} 2$ and $u p d 3$ were upregulated and these genes are enriched for histone H3 lysine 27 trimethylation (a target for PRC1 binding), indicating that PRC1 binds to and represses these target genes. Overexpression of upd caused an overgrowth phenotype similar to knockout of PRC1. Does reducing JAK-STAT signalling suppress tumour growth in a PRC1 mutant background? Expression of a dominant-negative form of the JAK-STAT receptor DOMELESS or the endogenous STAT inhibitor SOCS36E markedly reduced tumour growth, providing further confirmation that the overgrowth of PcG mutant imaginal discs is caused by the hyperactivation of the JAK-STAT pathway.

The identification of the PcG proteins as tumour suppressors in flies challenges our view of the role of PcG proteins in cancer. It will be interesting to determine the contexts in which PcG proteins have oncogenic and tumour suppressor roles, and how these findings translate to human cancers.

Meera Swami

ORIGINAL RESEARCH PAPERS Martinez, A.-M et al. Polyhomeotic has a tumor suppressor activity mediated by repression of Notch signaling. Nature Genet. 13 Sep 2009 (doi:10.1038/ng.414)| Classen, A.-K. et al. A tumor suppressor activity of Drosophila Polycomb genes mediated by JAKSTAT signaling. Nature Genet. 13 Sep 2009 (doi:10.1038/ng.445) 